\title{
PLANT DISEASE DIAGNOSIS AND SOLUTION SYSTEM BASED ON NEURAL NETWORKS
}

\author{
N. V. Megha Chandra Reddy \\ Research Scholar, \\ Department of Electronics and Communication Engineering, School of Engineering, CHRIST University, \\ Bangalore, Karnataka, India \\ nandyala.veera@btech.christuniversity.in

\section{K. Ashish Reddy} \\ Research Scholar, \\ Department of Electronics and Communication Engineering, School of Engineering, CHRIST University, \\ Bangalore, Karnataka, India \\ kancham.ashish@btech.christuniversity.in
}

Sushanth. G

Assistant Professor,

Department of Electronics and Communication Engineering, School of Engineering, CHRIST University, Bangalore, Karnataka, India sushanth.g@christuniversity.in

Sujatha. S

Assistant Professor,

Department of Electronics and Communication Engineering, School of Engineering, CHRIST University, Bangalore, Karnataka, India sujatha.s@christuniversity.in

\begin{abstract}
Plant diseases are one of the major factors affecting crop yield. Early identification of these diseases can improve productivity and save money and time for the farmer. This paper presents a novel technique to diagnose plant diseases using a mobile application. A Convolutional Neural Network (CNN) model was built and trained using MobileNetV2 architecture with the help of image processing techniques and transfer learning. A dataset comprising 87,000 images that contain 38 classes of diseases belonging to 14 different crops was used to train the model. The model achieved an accuracy of $98.69 \%$ and a loss of 0.5373 . A mobile application was built in Android Studio with the help of a trained model. The mobile application built works without a need for a remote server. The application can identify the disease, gives information regarding the identified disease and also suggests necessary remedies to tackle the disease.
\end{abstract}

Keywords: Convolutional Neural Network (CNN); Image Processing; MobileNet.

\section{The Main Text}

India is the world's largest and one of the oldest agriculture-dependent countries. More than half of the Indian population is involved in agriculture directly and indirectly. Though with such a large workforce, agriculture is contributing only $18 \%$ of the total Gross Domestic Product (GDP) [1]. The total factor productivity growth of India is below $2 \%$ per annum which is far lower than many other developing countries [2]. With the agricultural land shrinking exponentially, it's high time India employed productivity-improving strategies. One of the major hindrances of productivity is pest attacks. As high as $15.7 \%$ of losses of yield is due to plant diseases in India [3]. Thus, disease identification and monitoring are crucial to increase the yield of the crop.

Disease Identification and monitoring is an arduous task and if the cultivation is done in large areas, it becomes highly impossible to control the damage and prone to errors. Importantly, many farmers don't know the different kinds of diseases affecting the plants and what remedies to be employed. To counter this, many technologies have emerged to help the farmers in monitoring the field. Geographic Information Systems (GIS) were one of the most used technologies in monitoring the field. With the help of GIS, farmers can assess crop conditions and they also 
have data regarding the fertility levels of the soil [4]. Unmanned Aerial Vehicles (UAV) are the most talked-about technology for crop monitoring in the past decade. Using several high-tech types of equipment like near-infrared cameras, thermal sensors and other sensors [5]. The data obtained from UAVs is reliable and gives a detailed analysis of the crop obtained from different sensors. To achieve high precision results, Satellite Imaging is used. This technology can be employed to study vast areas of cultivation with the help of high-resolution images obtained from satellites [6]. Apart from GIS, UAVs and Satellite Imaging many other technologies are being employed to assist the farmers like the Internet of Things (IoT), merging datasets, farming software with the help of neural networks.

Though all these technologies have been producing great results, their usage in farmlands is very limited especially in countries like India, where most of the farmers have no idea of how to use and study these technologies. UAVs and Satellite Imaging is very expensive which can not be afforded by Indian farmers. Also, many of these technologies need an active network connection to the database which is not feasible in remote areas. So many of these technologies are only suitable for research work but not in practical scenarios.

To assist the farmers, in specific poor and middle-class Indian farmers, this paper suggests a mobile application that does crop diagnosis. In these modern times, almost everyone in India has a smartphone. Thus, this is the best optimal way to reach maximum users. The farmer need not know about the architecture employed in the application or the process of how it works. With zero extra knowledge required, farmers can easily use this application and can get instant results about the disease affected and the remedies that need to employed to counter the disease. There is no financial problem too as this application is free to use and also no issue with the network as this application even works offline. This mobile application is the best way of assisting farmers in crop monitoring and diagnosis.

The mobile application was built in two stages. Firstly, a Convolutional Neural Network (CNN) based model was built with the help of image processing techniques and transfer learning. Lastly, the built model was made into an application with the help of Android Studio. The built mobile application can identify 38 different classes of diseases in 14 different crops.

The remainder of this paper is organized as follows: Literature Survey is carried out in Section 2. Section 3 describes the methodology used in building the model and mobile application. The experimental setup is discussed in Section 4 and it is followed by the results in Section 5. Finally, this paper is concluded in Section 6.

\section{Literature Survey}

Mobile applications are being widely used by millions of people around the world. Masi et al. [7] presented a detailed framework for technology decision making for the development of mobile applications. They discussed technologies employed, programming languages used, platforms available, platform-specific software development kits and drivers involved. They even presented various problems faced and solutions to counter them.

From entertainment, health, business, productivity and everything else are achieved and improved with the help of mobile applications. Oinas-Kukkonen and Kurkela [8] discussed extensively developing a successful mobile application. The authors talked about different scenarios, applications and key design principles in developing an application for mobile devices. Islam and Mazumder [9] presented different areas of applications of mobile applications. The authors even discussed the effect of mobile applications on society and also talked about the limitations of mobile applications. Sunitha and Elina [10] discussed the impact of mobile applications in the education sector. The authors presented different kinds of apps used in education and subject-specific mobile applications and their advantages. Ventola [11] presented about mobile devices and apps used in the field of healthcare. The author mentioned different types of applications, their need at the point of care and how health care professionals use them. Choe et al. [12] discussed a real-time mobile application that can identify and classify different parrot species using CNN. The authors discussed the implementation of the mobile application, how the $\mathrm{CNN}$ model was built, feature extraction techniques employed and presented different experimental results.

Mobile applications are also being extensively used in the field of agriculture. Dehnen-Schmutz et al. [13] explored the farmer's usage of mobile phone technology in agriculture. The authors presented many surprising results that say a high percentage of farmers nearly $84 \%$ used mobile applications for farm management and many other farmers used it for real-time monitoring, data collection and experimental work. Johannes et al. [14] presented a novel image processing method for disease identification with the help of hot-spot detection and statistical inference methods using mobile devices. Toseef and Khan [15] developed a mobile application using a fuzzy inference system for crop diagnosis and it achieved an accuracy of $99 \%$.

Though different algorithms are used to build the model, CNNs are considered the best and accurate method of creating a model. Jiang et al. [16] demonstrated a real-time detection of leaf diseases in apples. The authors developed a model called INAR-SSD using a deep learning approach. The model achieved performed with a 78.8 
$\%$ mAP on the dataset used with a great speed of detection. Elhassouny and Smarandache [17] proposed a smart mobile application using CNN to recognize diseases in tomato leaves. The authors used a 7176 imaged dataset and built an application that can detect ten different types of diseases affecting tomato leaves. Esgario et al. [18] presented an application that identifies pests and diseases using deep learning in coffee leaves. The authors discussed how the android application can detect and classify diseases caused by biotic agents in coffee leaves with an accuracy of $97 \%$. Petrellis [19] presented a mobile application that runs without a server and can diagnose citrus diseases. The application achieved an accuracy of more than $90 \%$ in detecting citrus diseases. Rishiikeshwer et al. [20] built an application that can detect diseases in plants with an accuracy of $98 \%$ using CNN and image augmentation. The authors built a web application that requires a server to work.

Though many researchers presented a mobile application that can identify diseases, there are no papers that discuss a mobile application that can work without a remote server and can identifying diseases in 14 different crops with high accuracy. The main objective of this paper is to build a CNN model and a mobile application using the built CNN model.

\section{Methodology}

The proposed system is depicted in Fig. 1. The system can be broadly divided into two stages.

1. Creating a Model

2. Deploying the model

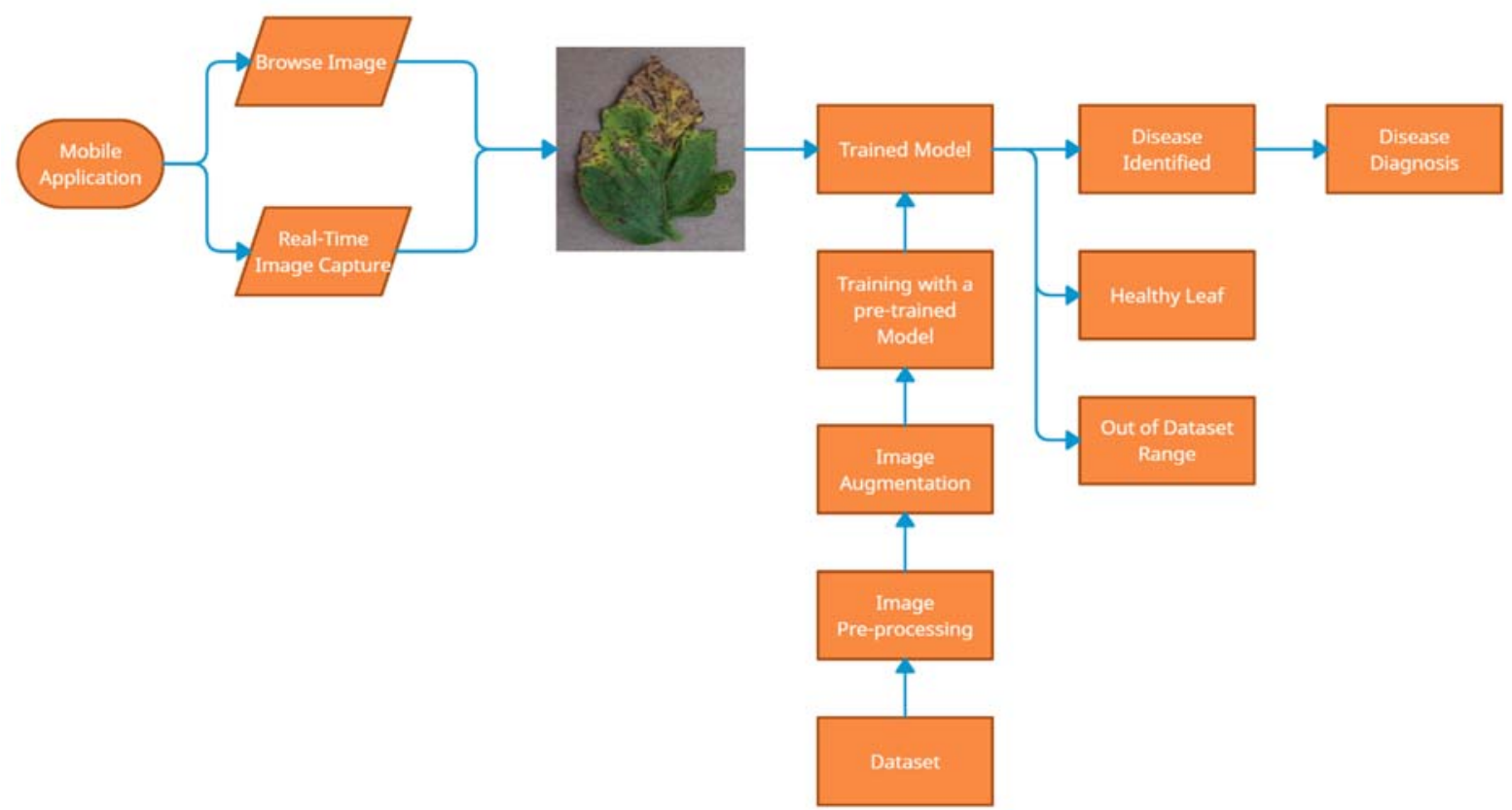

Fig. 1. Flowchart of the proposed system

\subsection{Creating a Model}

\subsubsection{Dataset}

The dataset used to train the model was espoused from an open-source platform [21]. It has a collection of 87000 leaf images from 14 different crops in jpeg format. Detailed information of the dataset is shown in Table 1. The dataset is partitioned into test, train and validation sets. The test set was created by picking out 20 to 30 images per disease in each crop and these selected images were removed from the main dataset. The remaining 85,851 dataset images were partitioned into train and validation sets in the ratio of 80:20 respectively.

\subsubsection{Image Processing}

Image pre-processing and image augmentation were the techniques utilized in training the model. As the dataset has minimal noise, no noise removal techniques were used. There was no change in the image format too as the model is trained using default jpeg images. Only image resizing is done as the default image size is $224 \mathrm{x} 224$ pixels for the model to be trained. 
Table 1. Dataset

\begin{tabular}{|c|c|c|c|c|}
\hline S. No & Crop Name & Disease Name & $\begin{array}{l}\text { Number of images in } \\
\text { the Train set }\end{array}$ & $\begin{array}{l}\text { Number of images in } \\
\text { the Validation set }\end{array}$ \\
\hline 1 & \multirow{4}{*}{ Apple } & Scab & 2016 & 504 \\
\hline 2 & & Black Rot & 1987 & 497 \\
\hline 3 & & Cedar Rust & 1760 & 440 \\
\hline 4 & & Healthy & 2008 & 502 \\
\hline 5 & Blueberry & Healthy & 1816 & 454 \\
\hline 6 & \multirow[b]{2}{*}{ Cherry } & Powdery Mildew & 1683 & 421 \\
\hline 7 & & Healthy & 1826 & 456 \\
\hline 8 & \multirow{4}{*}{ Corn } & Cercospora Leaf Spot & 1642 & 410 \\
\hline 9 & & Common Rust & 1907 & 477 \\
\hline 10 & & Northern Leaf Blight & 1908 & 477 \\
\hline 11 & & Healthy & 1859 & 465 \\
\hline 12 & \multirow{4}{*}{ Grape } & Black Rot & 1888 & 472 \\
\hline 13 & & Esca (Black Measles) & 1920 & 480 \\
\hline 14 & & Leaf Blight & 1722 & 430 \\
\hline 15 & & Healthy & 1692 & 423 \\
\hline 16 & Orange & Citrus Greening & 2010 & 503 \\
\hline 17 & \multirow[b]{2}{*}{ Peach } & Bacterial Spot & 1838 & 459 \\
\hline 18 & & Healthy & 1728 & 432 \\
\hline 19 & \multirow[b]{2}{*}{ Pepper } & Bacterial Spot & 1913 & 478 \\
\hline 20 & & Healthy & 1988 & 497 \\
\hline 21 & \multirow{3}{*}{ Potato } & Early Blight & 1939 & 485 \\
\hline 22 & & Late Blight & 1939 & 485 \\
\hline 23 & & Healthy & 1824 & 456 \\
\hline 24 & Raspberry & Healthy & 1781 & 445 \\
\hline 25 & Soybean & Healthy & 2022 & 505 \\
\hline 26 & Squash & Powdery Mildew & 1736 & 434 \\
\hline 27 & \multirow[b]{2}{*}{ Strawberry } & Leaf Scorch & 1774 & 444 \\
\hline 28 & & Healthy & 1824 & 456 \\
\hline 29 & \multirow{10}{*}{ Tomato } & Bacterial Spot & 1702 & 425 \\
\hline 30 & & Early Blight & 1920 & 480 \\
\hline 31 & & Late Blight & 1851 & 463 \\
\hline 32 & & Leaf Mold & 1882 & 470 \\
\hline 33 & & Septoria Leaf Spot & 1745 & 436 \\
\hline 34 & & Two-spotted Spider Mite & 1741 & 435 \\
\hline 35 & & Target Spot & 1827 & 457 \\
\hline 36 & & Yellow Leaf Curl Virus & 1961 & 490 \\
\hline 37 & & Mosaic Virus & 1790 & 448 \\
\hline 38 & & Healthy & 1926 & 481 \\
\hline Total & 14 & 38 & 68279 & 17572 \\
\hline
\end{tabular}

For the model to be performed well, a large size of data is needed while training the model. But in practical scenarios, satisfying such heavy data size requirements is not feasible. In such scenarios, data augmentation is done on the dataset to increase data size. In this model, image augmentation techniques like rescaling, 0.2 sheer and zoom ranging, horizontal flipping were done.

\subsubsection{Training}

To save time and to improve model performance with fewer data, pre-trained models were used with the help of transfer learning [22]. There are many pre-trained models available on the internet like Inception, ResNet, VGG and many others. As this paper main objective is to create a mobile application, MobileNetV2 [23] was used as the pre-trained model. The top and bottom layers of the MobieNetV2 were removed. The architecture employed to train the model using MobileNetV2 is shown in Fig. 2. 


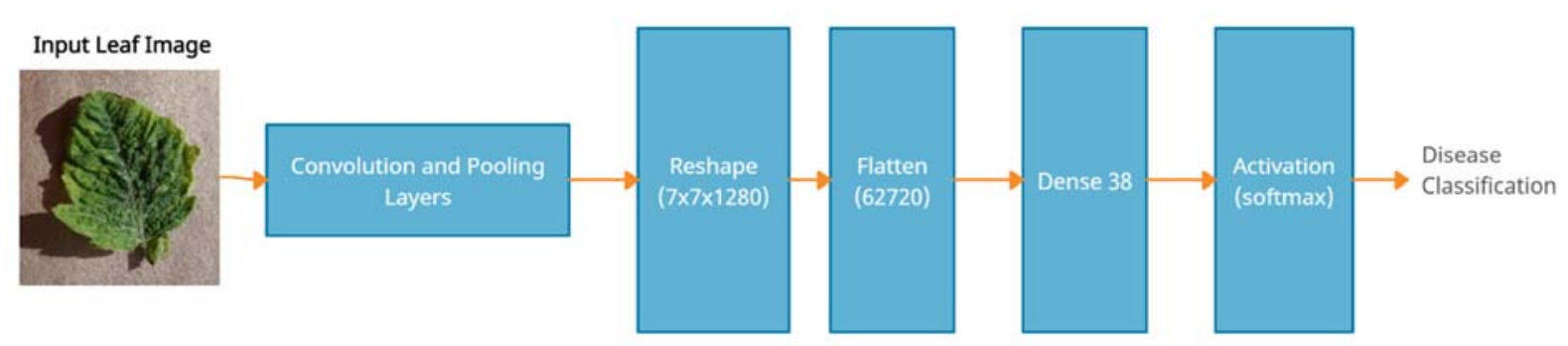

Fig. 2. The architecture of the MobileNetV2 model

\subsubsection{Optimizer}

Optimization is adjusting a mathematical expression. Optimizers are the methods that tune the model to reduce the losses. Adam [24] was the optimizer user in training this model. It works based on the formula shown in Eq. (1).

$$
J_{\min }=p-\eta \frac{M_{p}}{\sqrt{V_{p}}+\varepsilon} .
$$

where $p$ is a parameter, $\eta$ is the initial learning rate, $\mathcal{E}$ is a very small constant (to avoid divide by 0 ). $M_{p}$ and $V_{p}$ are derived from Eq. (2) and Eq. (3) respectively.

$$
\begin{aligned}
& M_{p}=\frac{m_{p}}{1-\beta_{1}} \\
& V_{p}=\frac{v_{p}}{1-\beta_{2}}
\end{aligned}
$$

where $m_{p}$ and $v_{p}$ are the mean and variance of the gradient respectively and $\beta_{1}, \beta_{2}$ are the bias correction constants.

\subsection{Deploying the Model}

The process of deploying a model as a mobile application is shown in Fig. 3.
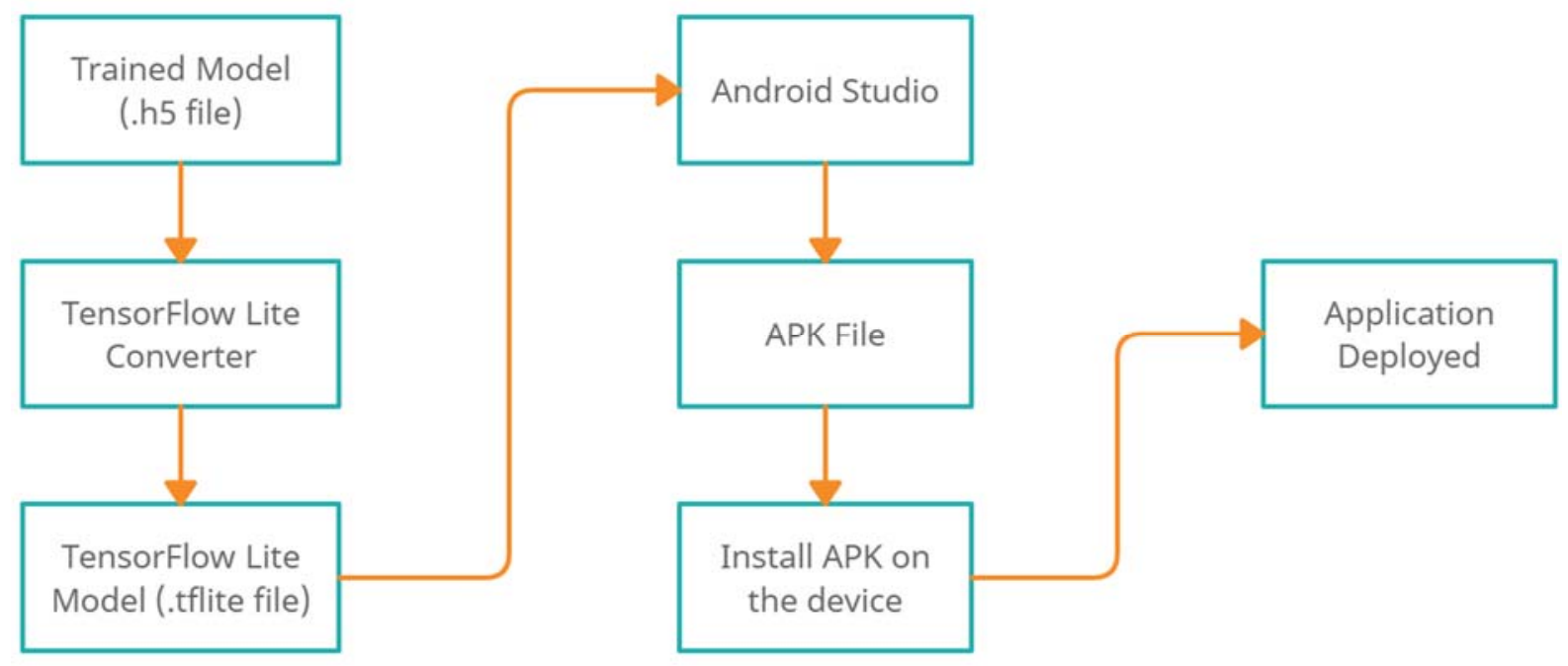

Fig. 3. Flowchart of deploying the model

The trained model directly can't be used in mobile devices due to its large size, high power consumption and incorrect formatting. Thus, the model needs to be lightweight and should have optimal power consumption. To achieve this the trained model was converted into a TensorFlow Lite file using a converter. The obtained tflite file is the speed and storage optimized version of the trained model.

The TensorFlow Lite Model was then passed on to Android Studio, an Integrated Development Environment (IDE) used for Android application development. In the Android Studio, the interface of the application was built using an interpreter and an Android Package File (APK) was created. This APK file can be shared through all Android devices. When this APK file is installed, the application is deployed on the device. 


\section{Experimental Setup}

CNN model was built using Google Colaboratory with a Tesla T4 GPU. Libraries like Keras, Pandas, NumPy, sklearn and PyTorch were utilized to train the model. Softmax is the activation function used. Adam and categorical cross-entropy were the optimizer and loss function used respectively. Hyperparameters for adam optimizer were set to default, $\eta=0.01, \beta_{1}$ and $\beta_{2}$ were set to 0.9 and 0.999 respectively. The model was trained for 50 epochs with a batch size of 32. The mobile application was built on Android Studio version 4.1.

\section{Results}

\subsection{CNN Model}

Results of the trained model can be seen in Fig. 4 and Fig. 5. The MobileNetV2 achieved a training accuracy of $98.69 \%$ and a training loss of 0.5373 .

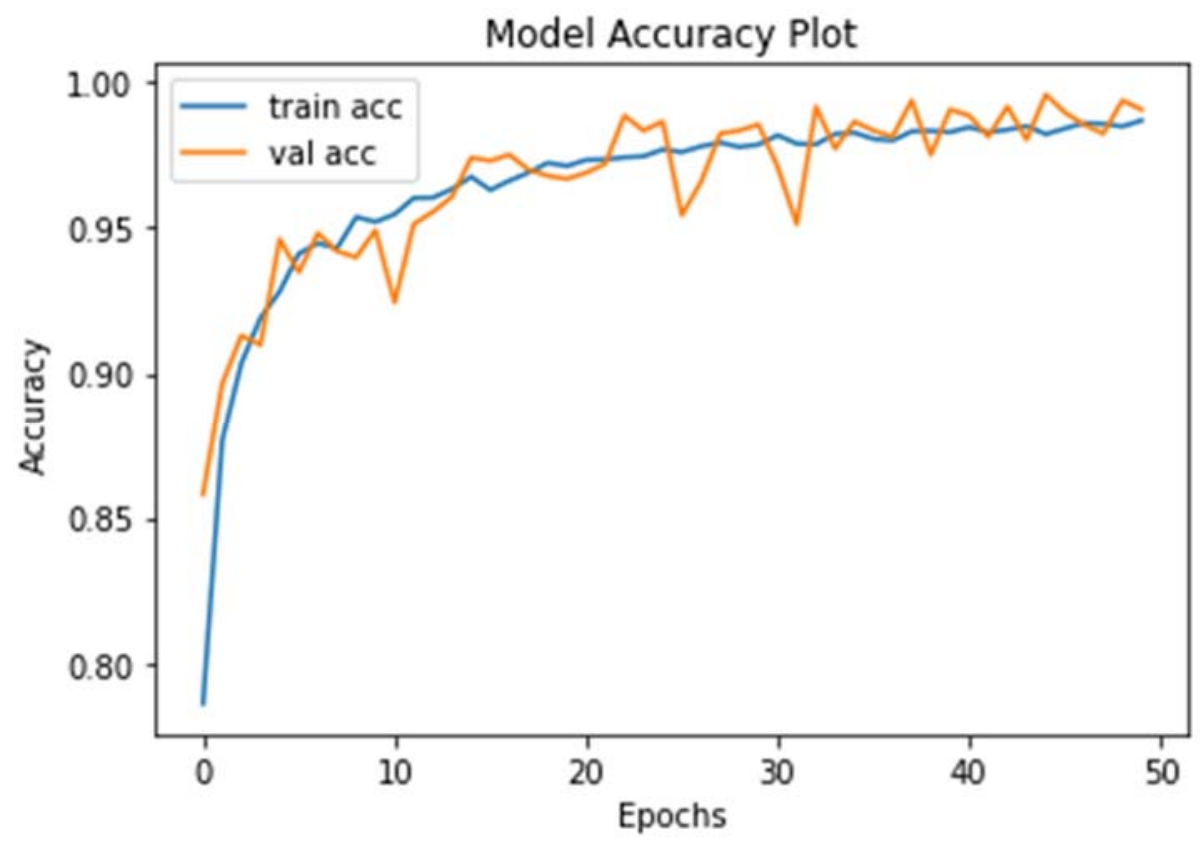

Fig. 4. Accuracy of the trained model

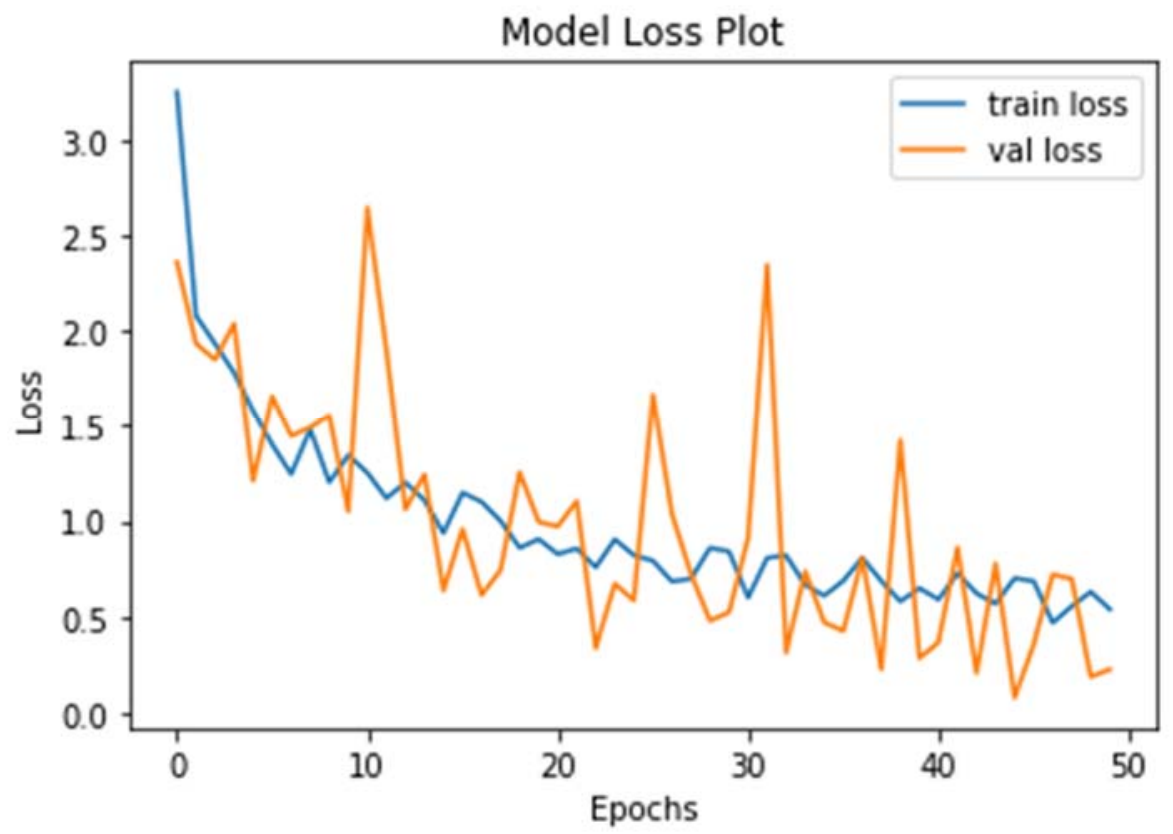

Fig. 5. Loss of the trained model 


\subsection{Mobile Application}

The home page of the mobile application is shown in Fig. 6 (a). There are two options available for the user. One is the user can upload a photo from the device (see Fig. 6 (b)) and the second one is the user can use real-time detection using a camera (see Fig. 6 (c)). If the input leaf is diseased, the 'name of the disease' is automatically displayed (see Fig. 6 (d)) with a 'know more' option which will redirect the user to the remedy page (see Fig. 6 (e)) or if the input leaf is healthy, it will be displayed 'Healthy Leaf' (see Fig. 6 (f)) or if the input leaf is not within the 38 classes of 14 crops, it will display an error message (see Fig. $6(\mathrm{~g})$ ).

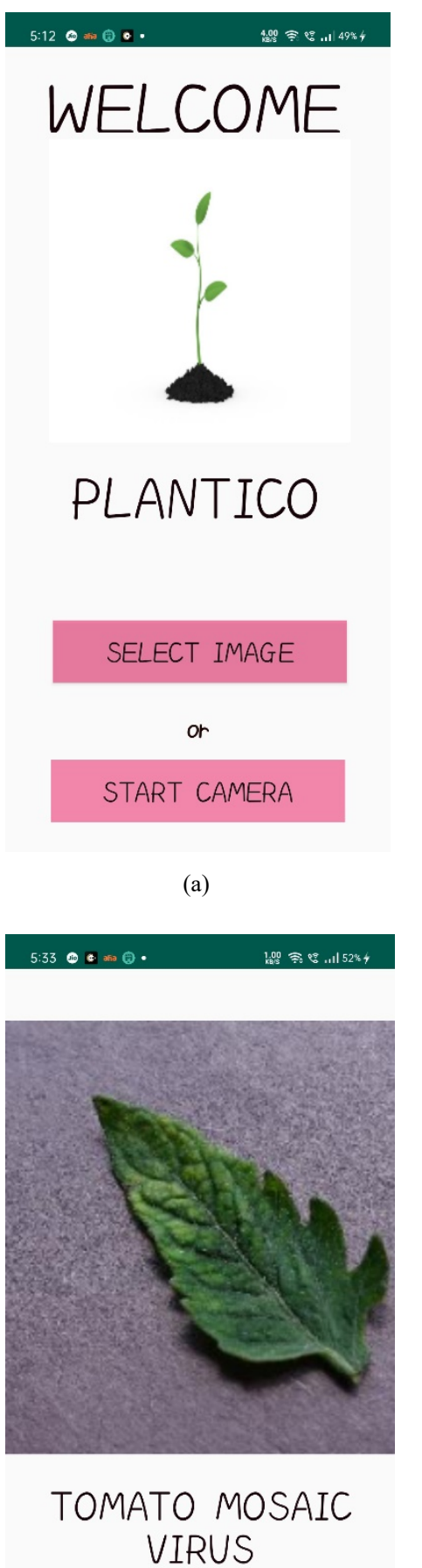

KNOW MORE

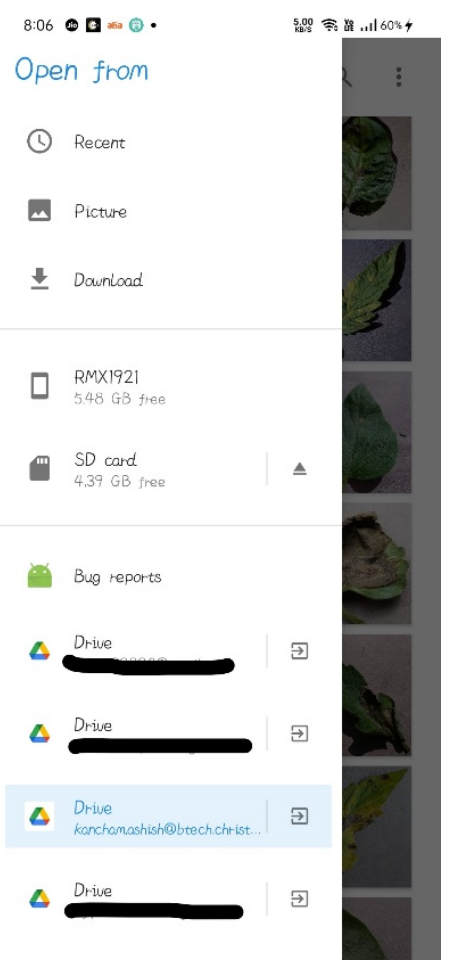

(b)

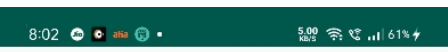

Tomato Mosaic Virus

\section{Symptoms:}

1. Infected leaves are distorted, with green and yellow mottling on the Leaves,

2. Plants are stunted to varying degrees,

\section{Treatment:}

There is no effective chemical control against Mosaic Virus, Later seeds can be soaked for 15 min in $100 \mathrm{~g} / \mathrm{l}$ solution of trisodium phosphate and then rinsed and dried.

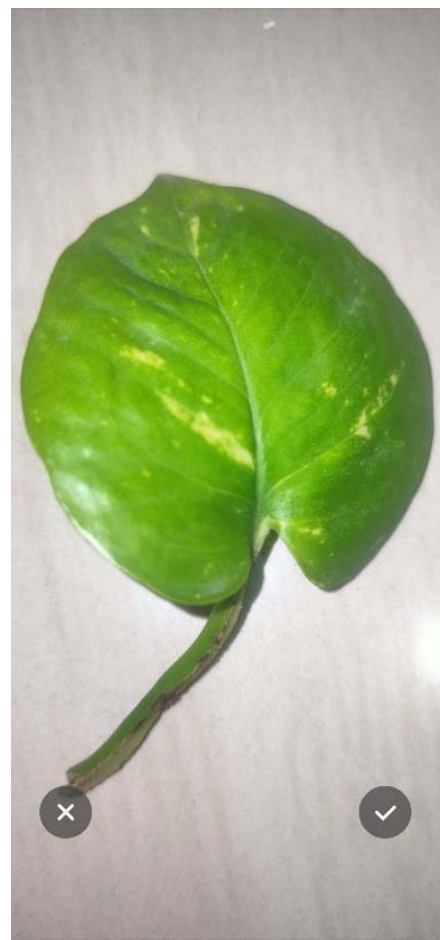

(c)
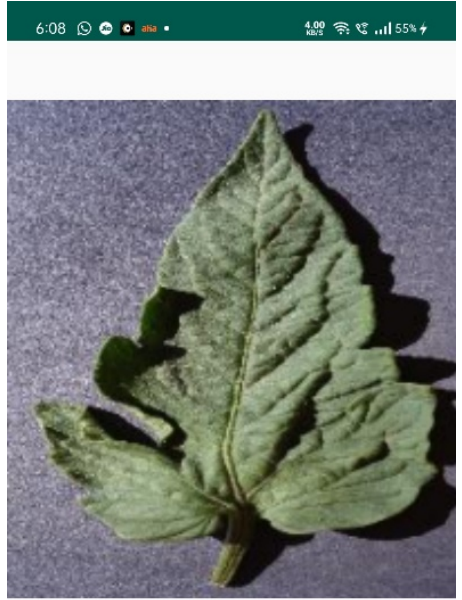

Healthy Image 


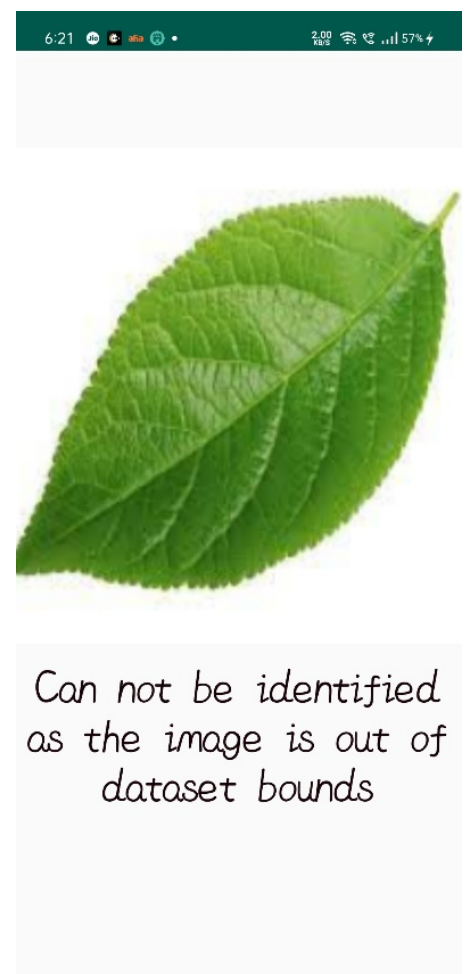

(g)

Fig. 6. Working of the mobile application

\section{Conclusion}

The main aim of this paper is to build a mobile application that can identify diseases and suggest remedies for the identified diseases. For this, firstly, a CNN model was built using MobileNetV2 architecture with the help of a dataset that contains 38 different classes of diseases belonging to 14 crops. The model is trained for 50 epochs with a batch size of 32 using adam optimizer. The model achieved an accuracy of $98.69 \%$ and a loss of 0.5373 . After obtaining the trained model, the model is converted into a TensorFlow lite file using a converter. Then the converted file was deployed in Android Studio, where the application interface was developed. An APK file was generated from the Android Studio and it can be shared to any android device and can be installed. The application developed is fast, lightweight and achieving great results. In future work, some optimization techniques will be employed on the application. Also, with sensors employed on the field, with the help of IoT, the sensor statistics will be integrated with the application thus providing customized data to the farmer.

\section{References}

[1] S. Sunder (2018). India economic survey 2018: Farmers gain as agriculture mechanisation speeds up, but more R\&D needed, The Financial Express, 2021. [Online]. Available: https://www.financialexpress.com/budget/india-economic-survey-2018-for-farmersagriculture-gdp-msp/1034266/. [Accessed: 03- Jul- 2021].

[2] Mahadevan, R., (2004). Productivity growth in Indian agriculture: The role of globalization and economic reform. Asia-Pacific Development Journal, 10(2), pp.57-72.

[3] Dhaliwal, G., Jindal, V. and Mohindru, B., (2015). Crop Losses due to insect pests: Global and Indian Scenario. Indian Journal of Entomology, 77(2), p.165.

[4] Dunaieva, I., Mirschel, W., Popovych, V., Pashtetsky, V., Golovastova, E., Vecherkov, V., Melnichuk, A., Terleev, V., Nikonorov, A., Ginevsky, R., Lazarev, V. and Topaj, A., (2019). GIS Services for Agriculture Monitoring and Forecasting: Development Concept. Advances in Intelligent Systems and Computing, pp.236-246.

[5] Ballesteros, R.; Ortega, J.F.; Hernández, D.; Moreno, M.A. (2014) Applications of georeferenced high-resolution images obtained with unmanned aerial vehicles. Part I: Description of image acquisition and processing. Precis. Agric. 15, 579-592.

[6] Mihai Valentin, Herbei \& Popescu, Cosmin \& Bertici, Radu \& Smuleac, Adrian \& Popescu, George. (2016). Processing and Use of Satellite Images in Order to Extract Useful Information in Precision Agriculture. Bulletin of University of Agricultural Sciences and Veterinary Medicine Cluj-Napoca. Agriculture. 73. 238. 10.15835/buasvmcn-agr:12442.

[7] Masi, Emiliano \& Cantone, Giovanni \& Calavaro, Giuseppe \& Mastrofini, Manuel \& Subiaco, Paolo. (2012). Mobile Apps Development: A Framework for Technology Decision Making. 10.1007/978-3-642-36632-1_4.

[8] Oinas-Kukkonen, Harri \& Kurkela, Virpi. (2003). Developing Successful Mobile Applications. Journal of Computer Science and Technology - JCST.

[9] Islam, Dr. MD Rashedul \& Mazumder, Tridib. (2010). Mobile application and its global impact. International Journal of Engineering \& Technology. 10. 72-78.

[10] Sunitha, R. and Elina, S., (2020). A Study on Mobile Applications in Education. IITM Journal of Management and IT, 11(1), pp.91-97. 
[11] Ventola CL. (2014) Mobile devices and apps for health care professionals: uses and benefits. P T;39(5):356-64. PMID: 24883008; PMCID: PMC4029126.

[12] Choe, D., Choi, E. and Kim, D., (2020). The Real-Time Mobile Application for Classifying of Endangered Parrot Species Using the CNN Models Based on Transfer Learning. Mobile Information Systems, 2020, pp.1-13.

[13] Katharina Dehnen-Schmutz, Gemma L. Foster, Luke Owen, Séverine Persello. (2016) Exploring the role of smartphone technology for citizen science in agriculture. Agronomy for Sustainable Development, Springer Verlag/EDP Sciences/INRA, 36 (2), pp.25. 10.1007/s13593-016-0359-9.

[14] A. Johannes, A. Picon, A. Alvarez-Gila, J. Echazarra, S. Rodriguez-Vaamonde, A. D. Navajas, and A. Ortiz-Barredo, (2017) Automatic plant disease diagnosis using mobile capture devices, applied on a wheat use case, Computers and Electronics in Agriculture, vol. 138, pp. 200-209.

[15] Toseef, M. and Khan, M., (2018). An intelligent mobile application for diagnosis of crop diseases in Pakistan using fuzzy inference system. Computers and Electronics in Agriculture, 153, pp.1-11.

[16] Jiang, P., Chen, Y., Liu, B., He, D. and Liang, C., (2019). Real-Time Detection of Apple Leaf Diseases Using Deep Learning Approach Based on Improved Convolutional Neural Networks. IEEE Access, 7, pp.59069-59080.

[17] Elhassouny, Azeddine \& Smarandache, Florentin. (2020). Smart mobile application to recognize tomato leaf diseases using Convolutional Neural Networks.

[18] Esgario, J., de Castro, P., Tassis, L. and Krohling, R., (2021). An app to assist farmers in the identification of diseases and pests of coffee leaves using deep learning. Information Processing in Agriculture,

[19] Petrellis, N., (2019). Plant Disease Diagnosis for Smart Phone Applications with Extensible Set of Diseases. Applied Sciences, 9(9), p.1952.

[20] S., R., Shriram, T., Raju, J., Hari, M., Santhi, B. and Brindha, G., (2020). Farmer-Friendly Mobile Application for Automated Leaf Disease Detection of Real-Time Augmented Data Set using Convolution Neural Networks. Journal of Computer Science, 16(2), pp.158166.

[21] "PlantVillage", Plantvillage.psu.edu, (2021). [Online]. Available: https://plantvillage.psu.edu/. [Accessed: 04- Jun- 2021].

[22] S. Pan and Q. Yang, (2010). A Survey on Transfer Learning, IEEE Transactions on Knowledge and Data Engineering, vol. 22, no. 10, pp. 1345-1359. Available: $10.1109 /$ tkde.2009.191.

[23] M. Sandler, A. Howard, M. Zhu, A. Zhmoginov and L. Chen, (2018). MobileNetV2: Inverted Residuals and Linear Bottlenecks, IEEE/CVF Conference on Computer Vision and Pattern Recognition, pp. 4510-4520, doi: 10.1109/CVPR.2018.00474.

[24] Kingma, Diederik \& Ba, Jimmy. (2014). Adam: A Method for Stochastic Optimization. International Conference on Learning Representations. Available: arxiv.org/abs/1412.6980. 